J. Clin. Chem. Clin. Biochem.

Vol. 19, 1981, pp. 1097-1101

\title{
Analyseverfahren für glykosidierte Hämoglobine Ein Methodenvergleich
}

\author{
Von Cornelia Maria Niederau und $H$. Reinauer \\ Lehrstuhl für klinische Biochemie und biochemische Abteilung des Diabetes-Forschungsinstituts,
Universität Düsseldorf
}

(Eingegangen am 2. Februar/9. Juni 1981)

Zusammenfassung: In 26 verschiedenen Blutproben wurden die glykosidierten Hämoglobine mit 6 verschiedenen Verfahren bestimmt und die Korrelation der Ergebnisse berechnet. Bei den chromatographischen Verfahren erzielt die Hochdruckflüssigkeitschromatographie die beste Auftrennung für die Bestimmung der Einzelkomponenten der glykosidierten Hämoglobine. Daher und wegen der besseren Präzision ist sie als leistungsfähige Referenzmethode anzusehen. Für. die Routinediagnostik wird derzeit das Mikrosäulenverfahren oder die Thiobarbitursäuremethode empfohlen. Die labile Aldiminform (Schiff'sche Base) stört die Analyse gewöhnlich nicht, denn die kann systematisch erfaßt oder eliminiert werden.

\section{Comparison of analytical methods for the estimation of glycosylated haemoglobins}

Summary: The glycosylated haemoglobins of 26 different blood samples were analysed by six different methods; the regression lines and the correlation coefficients were calculated from the analytical data. The best separation and estimation of the fast haemoglobin fractions were achieved by high performance liquid chromatography (HPLC). Therefore HPLC is regarded as the reference method for the analysis of fast haemoglobin fractions. Microcolumn chromatography or the thiobarbituric acid method are recommended for routine analysis in clinical laboratories. Since the labile aldimine (Schiff's base) can be calculated from the analytical data or destroyed by appropiate methods, this glycosylated haemoglobin fraction does not interfere with the results.

\section{Einführung}

Die Analyse der glykosidierten Hämoglobine gestattet eine retrospektive Beurteilung des Kohlenhydratstoffwechsels und hat sich auch in der Therapiefuihrung bei Diabetes mellitus mittlerweile klinisch bewährt.

Die Fraktion der glykosidierten Hämoglobine setzt sich zusammen aus den Hauptfraktionen Hämoglobin $\mathbf{A}_{1 \mathrm{a}}$, $\mathbf{A}_{1 b}$ und $\mathbf{A}_{1 \mathrm{c}}$, die in sich heterogen sind; gemeinsam ist ihnen ein charakteristisches chromatographisches und elektrophoretisches Verhalten (1-6, 9-12). Die heute verfügbaren Analyseverfahren erfassen entweder die $\mathrm{Ge}$ sàmtfraktion der schnellen Hämoglobine oder aber die Einzelfraktionen, wie das Hämoglobin $\mathrm{A}_{1 c}$. Daher werden die Normbereiche der glykosidierten Hämoglobine unterschiedlich angegeben. Diês erschwert die Vergleichbarkeit der erhobenen Daten. Zudem gibt es keine brauchbareñ Standards, so daß effektive interne und externe Qualitätskontrollen bislang nicht durchführbar sind.

Ein weiteres Problem resultiert aus Befunden, wonach in den zu analysierenden Proben neben der stabilen
Ketoaminform ein wechselnder Anteil der labilen Aldiminform (Schiff'sche Base) vorhanden sein kann $(9,10$, 15). Der größte Teil der gängigen Methoden vermag zwischen der Keto- und Aldiminform nicht zu unterscheiden und erfaßt sie undifferenziert gemeinsam. Hierdurch ist eine Unsicherheit entstanden, die es nunmehr in Anbetracht der experimentell erworbenen Kenntnisse des Glykosidierungsprozesses und der tatsächlichen Gegebenheiten bei Patienten auszuräumen gilt.

Das Ziel der vorliegenden Arbeit ist es daher, die verfügbaren Methoden durch Analyse derselben Proben miteinander zu vergleichen. Außerdem soll zur Analyse und klinischen Bedeutung der Aldiminform Stellung genommen werden.

\section{Methoden und Material \\ Probenvorbehandlung \\ Von 26 unterschiedlich gut eingestellten diabetischen Patienten wurden Blutproben gewonnen und mit EDTA ungerinnbar ge- macht. Dieses Blut wurde nach Hämolyse für vier der geprüften Methoden direkt eingesetzt. Die Hämolyse wurde durch ein Hämolysereagenz oder durch hypotone Behandlung der Erythro-}

0340-076X/81/0019-1097\$02.00

(C) by Walter de Gruyter \& Co. · Berlin - New York 
cyten und Einfrieren und Auftauen erreicht. Für die Hochdruckflüssigkeitschromatographie wurde das Hämolysat durch Millipore-Filter (Porendurchmesser: $5 \mu \mathrm{m}$ ) gegeben. Für die Thiobarbitursäuremethode wurden die Erythrocyten dreimal in physiologischer $\mathrm{NaCl}-\mathrm{Lösung}$ gewaschen, in doppeltem Volumen dest. Wasser aufgenommen, einmal eingefroren und aufgetaut und die Erythrocytenmembranen $30 \mathrm{~min}$ bei $10000 \mathrm{~g}$ sedimentiert. Der Überstand wurde in die Analyse eingesetzt, nachdem die Hämo globinkonzentration auf $50 \mathrm{~g} / \mathrm{l}$ eingestellt worden war. Die Proben blieben bis zur Analyse eingefroren.

\section{Analyseverfahren}

Konventionelle Säulenchromatographie nach Trivelli et al. (2)

In eine Säule $(0,9 \mathrm{~cm} \times 20 \mathrm{~cm})$ wurde Bio-Rex 70, 200$400 \mathrm{mesh}$, eingebracht und mit Phosphatpuffer $(0,042 \mathrm{~mol} / 1$, $\mathrm{pH}=6,74$, unter $\mathrm{Zusatz}$ von $\mathrm{KCN}, 10 \mathrm{mmol} / \mathrm{l}$ ) äquilibriert. 1-2 mg Hämoglobin, gelöst in diesem Puffer, wurden auf die Säule aufgetragen und mit dem Äquilibrierungspuffer unter 10 bar Druck und einer Flußrate von $0,6 \mathrm{ml} / \mathrm{min}$ entwickelt. Das Eluat wurde kontinuierlich in einer Durchflußküvette spektralphotometrisch bei $415 \mathrm{~nm}$ registriert. Es wurden Fraktionen von $2 \mathrm{ml}$ gesammelt. Nach Darstellung der Hämoglobinfraktionen $\mathrm{HbA}_{1 \mathrm{a}}, \mathrm{HbA}_{1 b}$ und $\mathrm{HbA}_{1 \mathrm{c}}$ wurde mit einem Phosphatpuffer $(0,15 \mathrm{~mol} / \mathrm{l}, \mathrm{pH}=6,42)$ die Hauptfraktion der Hämoglobine $\left(\mathrm{HbA}_{0}\right.$ bzw. $\mathrm{HbA}_{2}$ ) eluiert (Abb. 1). Nach kompletter Elution des Hämoglobins wurde die Säule mit dem niedermolaren Phosphatpuffer regeneriert. Dauer des chromatographischen Laufes: 4 Stunden.

Hochdruckflüssigkeitschromatographische Auftrennung $(11,12)$

Eine Stahlsäule $(0,4 \times 20 \mathrm{~cm})$ wurde mit Bio-Rex 70 , -200 mesh, gefüllt. Die Hochdruckflüssigkeitschromatographie wurde mit einem Kompaktgerät der Firma Hewlett-Packard (Modell HP 1084 B) bei einem effektiven Säulendruck von 20 bar durchgeführt. Es wurden zwei Puffer zur Elution eingesetzt:

Phosphatpuffer $1(0,042 \mathrm{~mol} / \mathrm{l}, \mathrm{pH}=6,74)$ unter Zusatz von KCN $(10 \mathrm{mmol} / \mathrm{l})$ und Phosphatpuffer $2(0,150 \mathrm{~mol} / \mathrm{l}, \mathrm{pH}=$ 6,42). Die Flußrate betrug 1,0-1,2 $\mathrm{ml} / \mathrm{min}$. Die Absorption des Eluats wurde bei $415 \mathrm{~nm}$ in einer Durchflußküvette gemessen. Der gekoppelte Rechner zeichnete die Elutionsprofile auf und druckte die Flächenintegrale prozentual aus (Abb. 2). Dauer einer Analyse: $20 \mathrm{~min}$

Mikrosäulenverfahren $(5,20,22)$

Käufliche Mikrosäulen (Bio-Rad, München; Isolab/Panchem, Kleinwallstadt) wurden nach den Vorschriften der Hersteller verwendet. Die Mikrosäulen können im Prinzip wiederverwendet werden nach vollständiger Regeneration und Entgasung des Harzes.

Thiobarbitursäure-Methode $(8,9)$

Die Analyse erfolgte nach den Angaben von Flückiger \& Winterhalter (8) sowie Fischer et al. (9).

\section{Spektroskopisches Verfahren}

Dieses Verfahren beruht auf den Arbeiten von Perutz et al. (7). Die Bindung von 2,3 Diphosphoglycerat (oder Inosithexaphosphat) an das Hämoglobin, wodurch eine charakteristische Spektralverschiebung zustande kommt (vgl. Abb. 3), wird durch Glykosidierung des $\beta$-endständigen Valin verhindert. Die Reagenziensätze können selbst angesetzt werden. Zudem wurde ein Reagenziensatz der Firma Abbott verwendet.

Elektroosmose $(10,23)$

Die elektrophoretische Trennung erfolgte auf Agarplatten bei pH 6,3 mit $60 \mathrm{~V}$ für $40 \mathrm{~min}$. Das Gel wurde $20 \mathrm{~min}$ bei $55^{\circ} \mathrm{C}$ getrocknet und bei $420 \mathrm{~nm}$ im Densitometer (Corning, Modell 720) ausgewertet (vgl. Menard et al. (10)).

\section{Ergebnisse}

Die Auftrennung und quantitative Bestimmung der schnellwandernden Hämoglobine mit Hilfe der konventionellen Säulenchromatographie, die bislang als Referenzmethode angegeben wird, ist in Abbildung 1 dargestellt. Die Fraktionen $\mathrm{HbA}_{1 \mathrm{a}}$ und $\mathrm{HbA}_{1 b}$ kommen nicht vollständig getrennt von der Säule, so daß eine gesonderte Auswertung dieser beiden Fraktionen nicht möglich ist. Daher werden $\mathrm{HbA}_{1 \mathrm{a}+\mathrm{b}}$ und $\mathrm{HbA}_{1 \mathrm{c}}$ als getrennte Fraktionen erfaßt. Variationskoeffizienten wurden für diese Methode nicht bestimmt.

Das hochdruckflüssigkeitschromatographische Verfahren (Abb. 2) zeigt eine qualitativ bessere Auftrennung als die Makrosäule nach Trivelli et al. (2). Dabei wird die Analyse in einem kürzeren Zeitraum (60 min einschließlich der Regeneration) als bei der Makrosäule durchgeführt. Der automatische Probengeber erleichtert zudem das Analyseverfahren. Bei wiederholter Messung derselben Probe

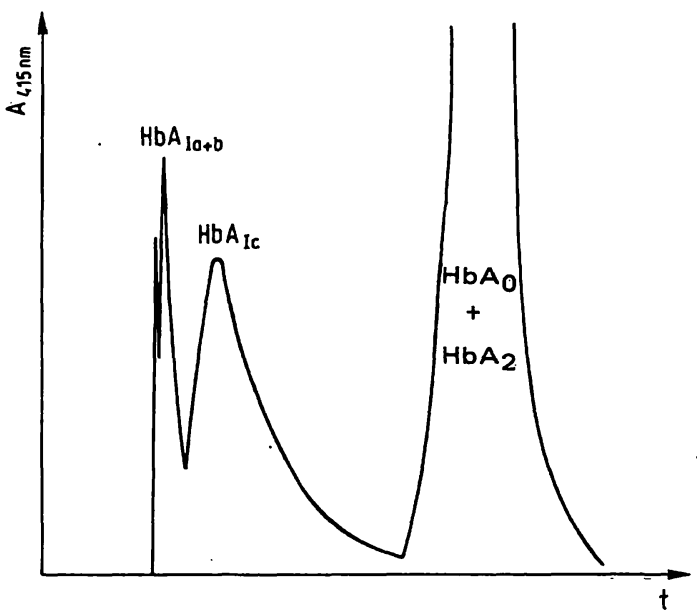

Abb. 1. Säulenchromatographische Auftrennung von Hämoglobin nach Trivelli et al. (2).

Puffer 1: 0,042 mol/1 Phosphat, $\mathrm{pH}=6,74$

Puffer 2: $0,150 \mathrm{~mol} / \mathrm{l}$ Phosphat, $\mathrm{pH}=6,42$

Säule $0,9 \times 20 \mathrm{~cm}$, Bio-Rex 70, 200-400 mesh.

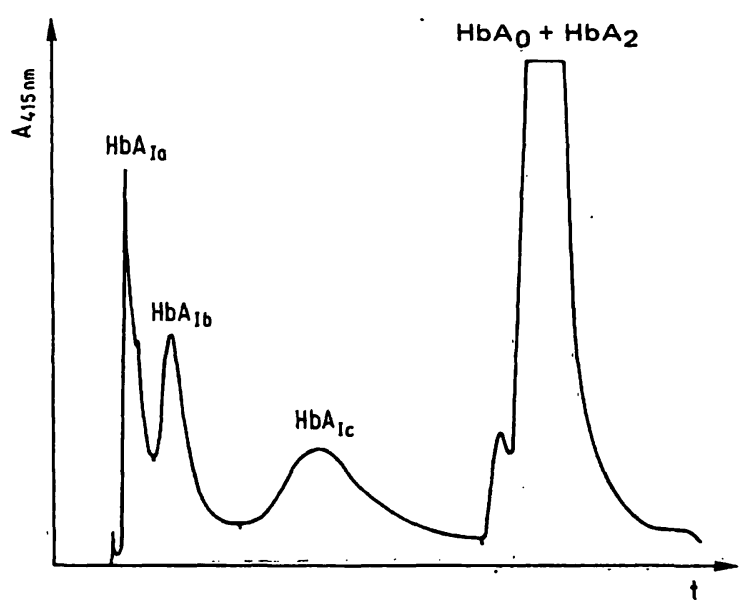

Abb. 2. Hochdruckflüssigkeitschromatographische Auftrennung von Hämoglobin auf Bio-Rex 70, - 200 mesh, Fluß $1,2 \mathrm{ml} / \mathrm{min}$, Druck 20 bar. 
ergab das hochdruckflüssigkeitschromatographische Verfahren einen Variationskoeffizienten für $\mathrm{HbA}_{1 \mathrm{a}}$ und $\mathrm{HbA}_{1 \mathrm{~b}}$ von $\mathrm{VK}=6,2 \%(\mathrm{n}=10)$ und $\mathrm{HbA}_{1 \mathrm{c}}$ von VK $=2,8 \%(n=10)$. Somit ist die HPLC-Methode als ein leistungsfähigeres Trennverfahren anzusehen und wird in der Folge als Referenzmethode eingesetzt. Die Analyse von $\mathrm{HbA}_{1 \mathrm{c}}$ mit der Makrosäule und der HPLC korrelierte mit $r=0,87(y=0,92 x-1,24)$. Ahnlich war die Korrelation der Gesamtfraktion $\left(\mathrm{HbA}_{1 \mathrm{a}, \mathrm{b}}\right.$ und $\mathrm{c}$ ) zwischen den beiden Verfahren mit $\mathrm{r}=0,88$ (Tab. 1).

Die Summe der schnellen Hämoglobine wird durch das standardisierte Mikrosäulenverfahren pauschal erfaßt, wobei die Auftrennungsqualität nicht zu beurteilen ist. Die Gesamtfraktion der schnellen Hämoglobine aus dem Mikrosäulenverfahren korreliert mit $r=0,97$ gut mit der Gesamtfraktion der schnellen Hämoglobine aus dem Makrosäulenverfahren (Abb. 4). Naturgemäß schlechter ist die Korrelation der Mikrosäulenwerte mit der $\mathrm{HbA}_{1 \mathrm{c}}{ }^{-}$

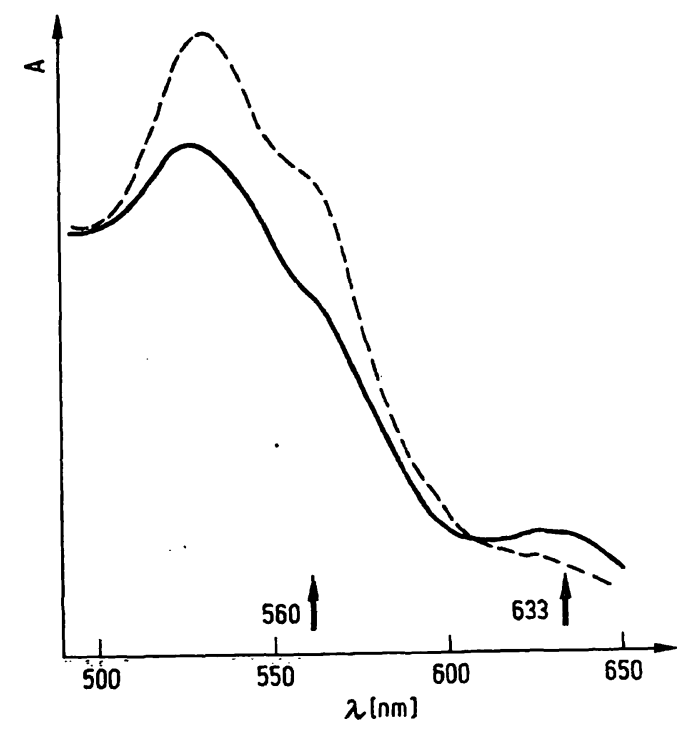

Abb. 3. Absorptionsspektrum von Hämoglobin vor (-- ) und nach $(-)$ Zugabe von Inosithexaphosphat als Grundlage der spektroskopischen Bestimmungsmethode.
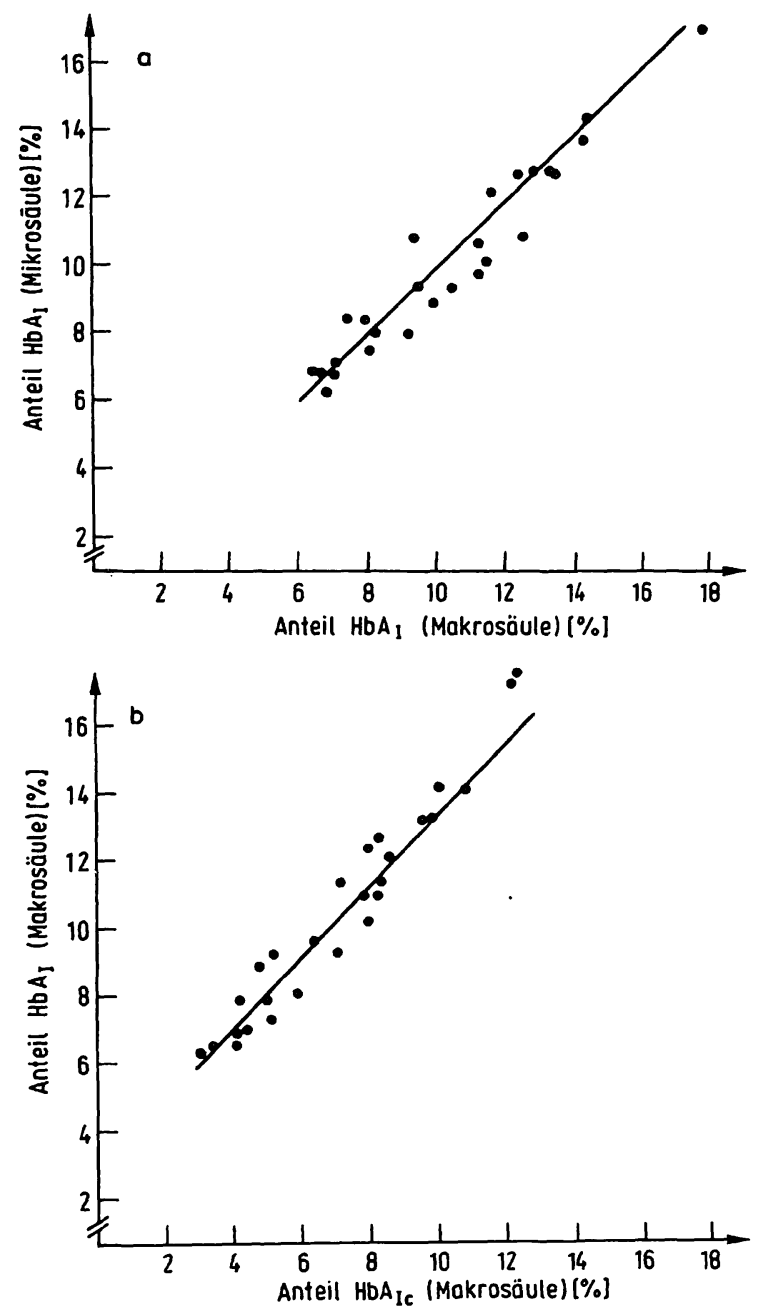

Abb. 4. Regressionsgerade und Korrelationskoeffizient zwischen dem säulenchromatographischen Verfahren (Makrosäule) und dem Mikrosäulenverfahren (a) sowie zwischen Makrosäule-Gesamtfraktion und $\mathrm{HbA}_{1} \mathrm{c}^{- \text {Fraktion (b). }}$

Tab. 1. Regressionsgeraden und Korrelationsköeffizienten zwischen den untersuchten Verfahren. $\phi=$ nicht berechnet.

\begin{tabular}{|c|c|c|c|c|c|}
\hline & HP̈ĹC & Mikrosäule & $\begin{array}{l}\text { Thiobarbitursäure- } \\
\text { Methode }\end{array}$ & Elektrophorese & Spektroskopie \\
\hline Makrosäule & $\begin{array}{l}r=0,88 \\
y=0,93 x-0,35\end{array}$ & $\begin{array}{l}r=0,97 \\
y=1,00 x+0,01\end{array}$ & $\begin{array}{l}x=0,89 \\
y=0,51 x+4,08\end{array}$ & $\begin{array}{l}r=0,88 \\
y=0,89 x+0,75\end{array}$ & $\phi$ \\
\hline HPLC & $\phi$ & $\begin{array}{l}r=0,92 \\
y=0,90 x+2,25\end{array}$ & $\begin{array}{l}r=0,87 \\
y=0,47 x+5,72\end{array}$ & $\begin{array}{l}r=0,92 \\
y=0,87 x+2,06\end{array}$ & $\phi$ \\
\hline $\begin{array}{l}\text { Thiobarbitursäure- } \\
\text { Methode }\end{array}$ & $\phi$ & $\begin{array}{l}r=0,89 \\
y=1,83 x+5,65\end{array}$ & $\phi$ & $\begin{array}{l}r=0,92 \\
y=1,62 x-5,24\end{array}$ & $\begin{array}{l}r=-0,20 \\
y=-60,62 x+26,36\end{array}$ \\
\hline Mikrosäule & $\phi$ & $\phi$ & $\begin{array}{l}x=0,88 \\
y=0,45 x+4,39\end{array}$ & $\begin{array}{l}x=0,91 \\
y=0,88 x+0,76\end{array}$ & $\begin{array}{l}r=-0,28 \\
y=-34,75 x+17,39\end{array}$ \\
\hline Makrosäule $\mathrm{HbA}_{1 \mathrm{c}}$ & $\phi$ & $\begin{array}{l}r=0,92 \\
y=0,82 x-1,73\end{array}$ & $\begin{array}{l}x=0,84 \\
y=0,41 x+1,62\end{array}$ & $\begin{array}{l}r=0,75 \\
y=0,58 x+0,75\end{array}$ & $\phi$ \\
\hline HPLC HbA $1 \mathrm{c}$ & $\begin{array}{l}x=0,98 \\
y=0,85 x-1,26\end{array}$ & $\begin{array}{l}x=0,92 \\
y=0,78 x+0,51\end{array}$ & $\begin{array}{l}r=0,86 \\
y=0,40 x+3,56\end{array}$ & $\begin{array}{l}x=0,90 \\
y=0,73 x+0,48\end{array}$ & $\phi$ \\
\hline
\end{tabular}


Fraktion aus der Makrosäulenchromatographie $(r=0,92$, $y=0,82 x-1,73)$.

Aus dem Vergleich der Analysen folgt, daß das Mikrosäulenverfahren bei sorgfältiger Einhaltung der Trennbedingungen, insbesondere der Temperatur $\left(21,5^{\circ} \mathrm{C}\right)$ ein zuverlässiges Ergebnis über die Glykosidierung der Hämoglobine liefert. Die Präzision des Mikrosäulenverfahrens liegt bei $V K=2,41 \%$ (intraassay) $(n=10)$.

Das kolorimetrische Verfahren nach Flückiger \& Winterhalter (8) erfaßt nur die Ketoaminform der glykosidierten Hämoglobine, da dieses Verfahren prinzipiell auf einem kolorimetrischen Fructosenachweis basiert (9). Aus den Arbeiten von Flückiger \& Winterhalter sowie Fischer et al. $(8,9)$ geht weiterhin hervor, daß außer dem $\beta$-endständigen Valin, das vorrangig für das chromatographische und elektrophoretische Verhalten der șchnellen Hämoglobine verantwortlich ist, auch weitere Aminogruppen ( $\epsilon$-Aminogruppen von Lysin) glykosidiert und die abgespaltene Fructose mit der kolorimetrischen Methode erfaßt werden. Daher sind von vornherein Unterschiede in den Meßdaten zu erwarten. In der Tat ist eine brauchbare Korrelation nur in einem mittleren Konzentrationsbereich zwischen $9-11 \% \mathrm{HbA}_{1}$ vorhanden. Unterhalb dieses Konzentrationsbereiches werden mit der Thiobarbitursäure-Methode zu niedrige, oberhalb zu hohe Werte gemessen (unveröffentlicht, vgl. auch Fischer et al. (9)). In unseren Proben lag der Korrelationskoeffizient beim Vergleich der Makrosäulenmethode mit der Thiobarbitursäure über den gesamten Konzentrationsbereich bei $r=0,84$, und es wurde eine Regressionsgerade von $y=0,41 x+1,62$ errechnet. Die Präzision der Thiobarbitursäure-Methode liegt in Abhängigkeit von der Konzentration des glykosidierten Hämoglobins bei $\mathrm{VK}=3,6$ (Normalbereich) und $\mathrm{VK}=4,2 \%$ bei $12,2 \%$ $\mathrm{HbA}_{1}$ (jeweils $n=10$ ). Ein Nachteil dieses Verfahrens ist die lange Analysendauer.

Ungünstiger erwies sich in unseren Händen das spektroskopische Verfahren, das weniger zeitaufwendig, bezüglich Reagenzienkosten billiger und theoretisch interessanter als die übrigen Verfahren ist. Die Analyse der 26 Proben ergab nämlich keine brauchbare Korrelation mit den säulenchromatographischen Verfahren und der Thiobarbitursäure-Methode (Tab. 1). Daher wurde dieses Analyseverfahren, dessen charakteristische Spektren in der Abbildung 3 dargestellt sind, nicht mehr weiterverwendet. Die Präzision der Methode (intraassay) war mit $\mathrm{VK}=1,2 \%(n=10)$ sehr gut.

Erwähnt werden soll schließlich ein elektrophoretisches Verfahren, bei dem die Gesamtfraktion der glykosidierten Hämoglobine durch Elektroosmose von der nicht glykosidierten Hb-Fraktion abgetrennt wird. Dieses Verfahren ist noch nicht auf dem Markt. Die Analyse der 26 Blutproben ergab eine gute Korrelation des elektrophoretischen Verfahrens mit der Mikrosäulenmethode $(r=0,91)$ und auch mit der Thiobarbitursäure-Methode $(r=0,92)$. Die Präzision des Verfahrens liegt bei VK $=10,8 \%(n=10)$.

Mit Ausnahme der Thiobarbitursäure-Methode erfassen alle Verfahren sowohl die Ketoamin- als auch die Aldiminform der glykosidierten Hämoglobine. Nach unseren Erfahrungen ist in der täglichen Analytik die Aldiminform nur bei Blutzuckerwerten über $3 \mathrm{~g} / 1 \mathrm{meßbar}$. In diesen Fällen ist es empfehlenswert, die Aldiminform zusätzlich zu bestimmen und sie als einen retrospektiven Kurzzeitparameter in die klinische Betrachtung einzubežiehen. Meßbare Erhöhungen des Aldiminanteils sind auch bei kurzzeitigem Ansteigen der Blutzuckerkonzentrationen über $3 \mathrm{~g} / 1 \mathrm{bzw}$. bei akuten Entgleisungen des Kohlenhydratstoffwechsels zu erwarten. Eine ausreichende klinische Evaluierung des Aldiminanteils ist bislang nicht erfolgt. Da die Aldiminform durch Dialyse von Hämoglobin, durch mehrfaches Waschen der Erythrocyten oder aber durch $\mathrm{pH}$-shift (30 min bei $\mathrm{pH} 5$ ) vollständig beseitigt werden kann, ist auch in diesen Fällen eine isolierte Bestimmung der stabilen Ketoaminform mit den chromatographischen und elektrophoretischen Verfahren möglich (24).

\section{Diskussion}

Die diagnostische Bedeutung der $\mathrm{HbA}_{1}$-Bestimmung bei der Stoffwechselfuihrung des Diabetikers ist unstreitig. Die bisherigen klinischen Erfahrungen haben gezeigt, daß hier ein valider Parameter für die Langzeitbeurteilung der Hyperglykämie (Dauer und Ausmaß) vorliegt $(24,25)$. Die verfügbaren Analyseverfahren werden unter verschiedenen Gesichtspunkten geprüft: Präzision, Vergleichbarkeit und Arbeitsaufwand. Ein abschließender Vergleich der vorliegenden Methoden auf Richtigkeit wird erst möglich sein, wenn stabile Standards für die interne und externe Qualitätskontrolle zur Verfügung stehen.

Beim gegenwärtigen Stand der Analytik scheiden die Makrosäulen sowie die Hochdruckflüssigkeitschromatographie als klinische Routineverfahren aus und werden im wesentlichen in der Forschung und als Referenzmethoden eingesetzt bleiben. $\mathrm{Da}$ auch die spektroskopische Methode, die zwar kostengünstig und wenig zeitaufwendig ist, aber in ihrer Richtigkeit unbrauchbare Werte liefert, ausscheidet, muß die tägliche klinische Analytik auf die Mikrosäule und auf die Thiobarbiturșäure-Methode, evtl. auf das elektrophoretische Verfahren, zurückgreifen. $\mathrm{Da}$ das von uns geprüfte elektrophoretische Verfahren noch nicht auf dem Markt ist und bisher wenige Daten vorliegen, kann hier eine genauere Bewertung nicht erfolgen. Hinsichtlich Mikrosäule und Thiobarbitursäure-Methode muß die Diskussion sachgerechter erfolgen. Der Einwand, nach dem das Mikrosäulenverfahren abżulehnen sei, weil es die labile Aldiminform miterfaßt, ist nicht haltbar. Die Aldiminform kann durch geeignete Verfahren zerstört oder isoliert als zusätzlicher Parameter erfaßt werden. In der klinischen Praxis 
kann die Aldiminform bei hochgradigen Hyperglykämien und akuten Entgleisungen des Kohlenhydratstoffwechsels eine Rolle spielen. Somit ist das Mikrosäulenverfahren durchaus - bei der erforderlichen Kenntnis der analytischen Gegebenheiten - gleichberechtigt anzuwenden, zumal es weniger personalintensiv und weniger zeitaufwendig ist als die Thiobarbitursäure-Methode.

Nach unseren Untersuchungen ergeben sich für die drei zu vergleichenden Methoden folgende Daten zur Präzi- sion: Mikrosäule VK $=2,4 \%$, Thiobarbitursäure-Methode $\mathrm{VK}=3,6$ bzw. 4,2\% und Elektrophorese $\mathrm{VK}=10,8 \%$. Ein Vergleich der Analysedaten ergibt, daß die HPLC nicht nur hinsichtlich der Auftrennung der drei Fraktionen, sondern auch hinsichtlich Präzision dem Mikrosäulenverfahren überlegen ist. Das weitere Ziel in der Analytik muß die Schaffung von brauchbaren Standards für die interne und externe Qualitätskontrolle sein. Auf dieser Basis muß dann eine breite klinische Evaluierung der Analysenverfahren erfolgen.

\section{Literatur}

1. Rahbar, S. (1968) Clin. Chim. Acta 22, 296-298.

2. Trivelli, L. A., Ranney, H. M. \& Lai, H.-T. (1971) N. Engl. J. Med. 284, 353-357.

3. Koenig, R. J., Peterson, C. M., Jones, R. L., Saudek, C., Lehrmann, M. \& Cerami, A. (1976) N. Engl. Med. 295, 417-420.

4. Koenig, R. J., Peterson, C. M., Kilo, C., Cerami, A. \& Williamson, J. R. (1976) Diabetes 25, 230-232.

5. Abraham, E. C., Huff, T. A., Cope, N. D., Wilson, J. B. Jr., Bransome, E. D. Jr. \& Huisman, T. H. J. (1978) Diabetes 27, 931-937.

6. Saibene, V., Brembilla, L., Bertoletti, A., Bolognani, L. \& Pozza, G. (1979) Clin. Chim. Acta 93, 199-205.

7. Perutz, M. F., Sanders, J. K. M., Chenery, D. H., Noble, R. W., Pennely, R. R., Fung, L. W.-M., Ho, C., Giannini, I., Pörschke, D. \& Winkler, H. (1978) Biochemistry 17, 3640-3652.

8. Flückiger, R. \& Winterhalter, K. H. (1976) FEBS Letters 71, $356-360$.

9. Fischer, R. W., De Jong, C., Voigt, E., Berger, W. \& Winterhalter, K. H. (1980) Clin. Lab. Haemat. 2, 129-138.

10. Menard, L., Dempsey, M. E., Blankstein, L. A., Aleyassine, M., Wacks, M. \& Soeldner, J. S. (1980) Clin. Chem. 26, $1598-1602$.

11. Cole, R. A., Soeldner, J. S., Dunn, P. J. \& Bunn, H. F. (1978) Metabolism 27, 289-301.

12. Davis, J. E., McDonald, J. M. \& Jarrett, L. (1978) Diabetes 27, 102-107.
13. Gabbay, K. H., Hasty, K., Beslow, J. L., Ellison, R. C., Bunn, H. F. \& Gallop, P. M. (1977) J. Clin. Endocrinol. Metab. 44, 859-864.

14. Holmquist, W. R. \& Schroeder, W. A. (1964) Biochim. Biophys. Acta 82, 639-641.

15. Holmquist, W. R. \& Schroeder, W. A. (1966) Biochemistry 5, 2489-2503.

16. Bookchin, R. M. \& Gallop, P. M. (1968) Biochem. Biophys. Res. Commun. 32, 86-93.

17. Bunn, H. F., Haney, D. N., Gabbay, K. H. \& Gallop, P. M. (1975) Biochem. Biophys. Res. Commun. 67, 103-109.

18. McDonald, M., Shapiro, R., Bleichman, M., Solway, J. \& Bunn, H. F. (1978) J. Biol. Chem. 253, 2327-2332.

19. Shapiro, R., McManus, M. J., Zalut, C. \& Bunn, H. F. (1980) J. Biol. Chem. 255, 3120-3127.

20. Welch, S. G. \& Boucher, B. J. (1978) Diabetologia 14, 209 211.

21. Dixon, H. B. F. (1972) Biochem. J. 129, 203-208.

22. Kynoch, P. A. M. \& Lehmann, H. (1977) Lancet $I I, 16-18$.

23. Allen, R. C., Stastny, M., Hallett, D. \& Bimmons, M. A. (1979) in: Electrophoresis (B. J. Radola, ed.) De Gruyter, N.V. 1980 . p. $663-670$.

24. Niederau, C. M., Potthoff, S., Gries, F. A. \& Reinauer, H. (1980) Lab. Med. 4, 9-14.

25. Niederau, C. M., Potthoff, S. \& Reinauer, H. (1981) Z. Geburtsh. Perinat. $185,265-269$.

C. M. Niederau

Diabetes-Forschungsinstitut an der Universität Düsseldorf Auf'm Hennekamp 65

D-4000 Düsseldorf 1 
